\title{
Green marketing en empresas floricultoras de la Sabana de Bogotá
}

\section{Green marketing in floricultural companies in Sabana de Bogota}

\author{
Juan Carlos Osma Rozo ${ }^{1}$ \\ Julián Francisco Figueroa Espinel ${ }^{2}$ \\ Marlybell Ochoa Miranda ${ }^{3}$ \\ DOI: 10.29151/hojasyhablas.n18a3
}

\section{Resumen}

La floricultura colombiana, desarrollada desde la década de los 60, ha presentado un alto crecimiento a partir de un modelo de agricultura intensiva. Colombia es uno de los países con mayores exportaciones de flores de corte en el mundo, situación que hace necesario identificar el uso de estrategias de green marketing por parte de las empresas floriculturas en la sabana de Bogotá, con el fin de conocer los beneficios para la construcción de ventajas comparativas y competitivas en mercados altamente exigentes. Este estudio se realizó a través de un enfoque descriptivo de tipo cualitativo, a través de entrevistas a los productores del territorio sabanero sobre la importancia que le dan las empresas floricultoras a la aplicabilidad del concepto de mercadeo verde, enfatizando en cómo utilizan este concepto al momento de establecer las estrategias relacionadas con el mix de marketing y cómo informan a clientes y consumidores sobre su aplicabilidad dentro de la empresa, como valores diferenciadores en mercados nacionales e internacionales.

Palabras Clave: Medio ambiente; mercadeo verde; factores diferenciadores; floricultura.

\begin{abstract}
Colombian floriculture, developed since the 1960s, has shown high growth based on an intensive agriculture model. Colombia is one of highest exports of cut flowers on the world, that is the reason to identify the use of green marketing strategies by floriculture companies in the Sabana of Bogotá, allowing to know the benefits for the construction of comparative and competitive advantages in this market. The study was carried out through a descriptive approach of qualitative type, doing interviews with the producers of the territory, inquiring about the importance that flower growers give to the applicability of the concept of green marketing, and emphasizing how they use this concept in order to establish strategies related to the marketing mix, and at the same time how they inform customers about their applicability within the company, as differentiating values in national and international markets.
\end{abstract}

Keywords: Environment; green marketing; differentiating values; floriculture.

\footnotetext{
${ }^{1}$ Ingeniero Comercial. Magister en Dirección de Marketing. Docente investigador Fundación Universitaria Monserrate. Líder del grupo de investigación Gestión de Negocios y Mercados - GENEME. E-mail: josma@unimonserrate.edu.co. ORCID: 0000-0002-9446-6623

${ }^{2}$ Ecólogo. Magíster en Gestión Ambiental. Docente investigador Universidad Santo Tomas de Aquino. Integrante del Grupo de Investigación en Responsabilidad Social y Ambiental - GIRSA. E-mail: julianf.figueroa@gmail.com. ORCID: 0000-0003-1879-6839

${ }^{3}$ Ecóloga. Especialista en Derecho Ambiental. Docente investigadora Fundación Universitaria Monserrate. E-mail: marly_ochoa@yahoo.com
} 


\section{Introducción}

El concepto de Green marketing aparece en 1970 con investigadores estadounidenses que buscaron hacer contribuciones al desarrollo del marketing, pero con una perspectiva ecológica, preocupándose por el medio ambiente y un comportamiento ecológico. (Sixto y Salgueiro, 2008). Queriendo satisfacer las necesidades de los consumidores con conciencia ecológica, las empresas han iniciado procesos de producción encaminados a lograr un desarrollo sustentable de la economía y la sociedad, fundamentado en el enfoque social, entendido como las acciones hechas por instituciones sin fines de lucro, fomentando ideas y comportamientos de respeto al ambiente entre ciudadanos y sus agentes, así mismo el enfoque empresarial donde las organizaciones adoptan un sentido de compromiso con el medio ambiente, satisfaciendo las necesidades de los clientes y consumidores para que las metas se cumplan y sobre todo minimicen los impactos negativos al ambiente.

Así pues, la floricultura que se desarrolla en Colombia, y en particular en la Sabana de Bogotá, debería estar acompañada de una serie de actividades que incentiven una alta sensibilidad por el cuidado del medio ambiente y ayuden a las empresas a comprender la problemática como consecuencia del proceso productivo en donde cada paso del proceso debe tener el mínimo análisis para la conservación ambiental. Dentro de los aspectos más dañinos, y que se pueden solucionar con estrategias de green marketing, se encuentran el uso de productos que presentan un bajo nivel de biodegradación, el uso de materiales en el proceso productivo con residuos peligrosos, y el gasto de energía y materiales, que en su producción / distribución / consumo son contaminantes.
La existencia del mercadeo verde o ecológico en Colombia es un tema que apenas inicia. Algunas empresas pequeñas y medianas han adoptado esta tendencia realizando prácticas para el desarrollo de nuevos productos, y el gobierno colombiano no ha sido ajeno a esta tendencia, generando programas de sostenibilidad y fomento de prácticas ecológicas empresariales. Sin embargo, hay una asimetría en el proceso de certificación ecológica frente a los mercados internacionales (Echeverry, 2010).

El Plan Nacional de Desarrollo 2002-2006 "Hacia un Estado Comunitario" estableció como uno de los aspectos principales el programa de Sostenibilidad Ambiental de la Producción Nacional, impulsando la incorporación de la dimensión ambiental en los procesos de planeación sectorial, con énfasis en las Evaluaciones Ambientales Estratégicas, (EAEs), y el fortalecimiento del actual proceso de licenciamiento ambiental, donde se busca incorporar consideraciones y criterios ambientales en el proceso de planeación, garantizando el equilibrio entre sostenibilidad ambiental y desarrollo socioeconómico. Tema que se puede promover a través del Ministerio de Ambiente, Vivienda y Desarrollo Territorial (Ministerio de Ambiente, Vivienda y Desarrollo Territorial, 2006).

Las tendencias del mercado exigen prácticas corporativas en las que se fomente el desarrollo sostenible a corto, mediano y largo plazo, con implicancias en las prácticas a lo largo de toda la cadena de valor de las empresas, y con ello el desarrollo de actividades que incluyen la modificación del producto o servicio, cambios en los procesos productivos, en su distribución, modificaciones al empaque y actividades promocionales y de publicidad, capacitación de los colaboradores, entre otras. Esto lleva a plantear estrategias del mix de 
marketing, encaminadas al cumplimiento de los objetivos corporativos, sirviendo como herramienta para la generación de ventajas competitivas y siendo ambientalmente responsables (Pennano y Diaz Artola, 2014). Por esta razón, la investigación partió del interrogante ¿Cómo se están implementando estrategias de green marketing en las empresas floricultoras de la sabana de Bogotá y de qué manera se están viendo favorecidas?

El proyecto buscó identificar la influencia en productores y consumidores de la implementación de estrategias encaminadas a la conservación del medio ambiente desde el mismo proceso productivo, seguido de la comercialización de productos y/o servicios, integrando acciones encaminadas a lograr la captación de clientes y consumidores mediante un mix de marketing que resalte los beneficios del producto a través de valores diferenciadores que contribuyen a proteger y propender por un equilibrio entre lo social, económico y ambiental.

Con el paso de los años, el factor medioambiental ha tomado mayor importancia para los consumidores actuales, influyendo en la toma de decisiones frente a una compra, convirtiéndose el mercadeo verde en un elemento de vital importancia para el planteamiento de las estrategias empresariales, creando una diferenciación frente a la competencia y eliminando algunos impactos negativos de la empresa. El desarrollo de una gestión eco amigable no solo se evidencia en los beneficios para el planeta, sino en el mejoramiento de la imagen de marca, el grado de confiabilidad de clientes internos y externos, la reducción de costos, la optimización de procesos que incrementan la eficacia y productividad, el mejoramiento de imagen de la empresa frente a sus stakeholders, el mejoramiento de las relaciones con entidades gubernamentales, una mayor motivación y compromiso de los trabajadores, y la reducción del impacto en el entorno (Darse, s.f).

\section{Metodología}

Con el fin de identificar el uso de estrategias de Green marketing por parte de las empresas floriculturas de la sabana de Bogotá, y conocer los beneficios proporcionados para la construcción de ventajas competitivas, se adelantó el presente estudio de tipo descriptivo que especifica propiedades, características y rasgos importantes del fenómeno analizado (Hernández, Fernández, y Baptista, 2010); en este caso, relacionado con el conocimiento y uso del green marketing en las empresas floricultoras de la sabana de Bogotá.

A través de un enfoque cualitativo, y teniendo como universo a las empresas floricultoras de la sabana de Bogotá, se realizó un muestreo no probabilístico por conveniencia. La investigación fue realizada en tres fases: Fase I "Levantamiento de información secundaria”, en donde se planteó una revisión teórica para detallar los avances que se han tenido en el planteamiento y desarrollo de estrategias basadas en green marketing, en especial en el sector agropecuario, siendo aún más específico en las empresas floricultoras, a través de un proceso sistemático y crítico dada la necesidad de crear conciencia tanto en oferentes como en demandantes en actividades encaminadas a cuidar del medio ambiente.

En la fase II "Trabajo de campo y análisis de información", se hizo contacto directo con las empresas floricultoras de la sabana de Bogotá, con el propósito de identificar conocimiento sobre green marketing e implementación de estrategias desarrolladas, sus aplicaciones en cada componente de la mezcla de mercadotecnia y los beneficios que estas generan como parte de sus procesos 
organizacionales. En esta fase se utilizó una entrevista semiestructurada como técnica de recolección de información, donde se pudo identificar el desconocimiento frente al concepto y aplicación en el proceso de producción y comercialización de flores. La fase III "Socialización de resultados" consistió en la difusión de los hallazgos en comunidades académicas y del sector productivo, mostrando los aciertos y desaciertos frente a la implementación de estrategias de mercadeo ver$\mathrm{de}^{4}$ en el sector floricultor.

El mercadeo verde, integrado por todas las actividades que buscan generar y facilitar los intercambios destinados a satisfacer las necesidades humanas o deseos con el mínimo impacto negativo hacia el medio ambiente natural (Dubey, 2008), se convierte en un proceso que debe abarcar todas las fases de la vida útil del producto, desde la obtención de materia prima hasta la disposición final de residuos o de reutilización. En el primer Congreso Internacional de Mercadeo Sustentable realizado en la ciudad de Caracas en el año 2011 se hizo referencia a que esta tendencia busca establecer conciencia en la implementación de estrategias por parte de las empresas, promoviendo el consumo ético (Caguao, 2011). Por otro lado, se resalta que el mercadeo verde no puede verse como una "tendencia" sino que debe asumirse como una "forma de vida", una filosofía de trabajo (Palazón, 2009).

El green marketing se convierte en protagonista de las estrategias de comunicación empresarial, buscando que las organizaciones sean socialmente responsables y generando posicionamiento en los consumidores, debido a que estos están demandando productos donde se destacan valores encaminados a la sostenibilidad ambiental y social, pasando el mercadeo verde de ser una tendencia a un requisito que los consumidores no quieren negociar. (Paternina, 2011)

\section{Green marketing en el ámbito nacional e internacional}

En 1992 en la Conferencia de las Naciones Unidas sobre Medio Ambiente y Desarrollo los gobiernos hicieron suyo el concepto de desarrollo sustentable, el cual hace referencia a lograr los objetivos económicos sin poner en riesgo los recursos naturales para las generaciones futuras. Siendo primordial para el cumplimiento de este objetivo la atención al consumidor, el cual comienza a desarrollar lo denominado como consumo sustentable, creando conciencia de la importancia y las consecuencias que tienen para el ambiente las decisiones relacionadas con él consumo, distinguiéndose por su preocupación por el medio ambiente en su comportamiento de compra y siendo este el nacimiento de un nuevo segmento de consumidores (Salgado et al., 2009).

En la Conferencia Anual de la American Marketing Asociation (AMA) de 1993, se estableció, por primera vez, una sesión especial dedicada al marketing ecológico, se consolidó una nueva concepción sobre empresa más allá de producir, teniendo responsabilidad a través de factores como preservación del medio ambiente y la racionalidad en el consumo de energía. Se orienta la empresa ante las exigencias de un desarrollo sostenible y satisfacción de la demanda de los diferentes grupos de interés denominados stakeholders (Sixto y Salgueiro, 2008).

\footnotetext{
${ }^{4}$ Mercadeo verde, conocido con el nombre de mercadeo ambiental, sustentable, medioambiental o ecológico (Chamorro, 2001). Naciendo en los 80 como el mercadeo de productos seguros para el medio ambiente incluyendo actividades de modificación al producto, proceso productivo, empaque y comunicación, manifestando de esta forma responsabilidad, conciencia y sensibilidad ecológica (Caguao, 2011).
} 
Es así como, las organizaciones deben buscar tecnologías más limpias, involucrar las normas internacionales como la ISO 14001 relacionada con el sistema de gestión ambiental, para la producción de bienes para un consumidor eco amigable, respondiendo a las preguntas ¿cómo ha sido elaborado el producto?, ¿de dónde vienen las materias primas?, ¿qué tan limpio es el proceso productivo?, ¿qué sucede con el producto al final de su ciclo de vida? Ya en una tercera etapa, para el año 2000, significó la aplicación de tecnologías más avanzadas, regulaciones más estrictas por parte de los gobiernos y una mayor conciencia ambiental (Hammann, S.f).

Como consecuencia del green marketing no solo está la creación de productos para la compra y venta, sino la inclusión de leyes gubernamentales, campañas en pro de cuidar los recursos naturales, manejo de residuos a nivel empresarial como en el hogar, restricción de la circulación de vehículos durante algunos días de la semana, cacería y el tráfico ilegal de animales, como muestra de algunas acciones que buscan controlar el daño ambiental generado por el ser humano en el desarrollo de sus actividades diarias. Sin embargo, falta hacer mucho más a través del mercadeo verde, como la creación de servicios especializados para empresas que desarrollen este tipo de estrategias relacionadas con el mercadeo verde, la motivación hacia una conciencia ambiental por parte de los consumidores, desarrollo de tecnologías que reemplacen aquellas que son obsoletas, entre otras (Berrio, 2016).

El green marketing se perfila como una de las estrategias clave para el mundo de los negocios del nuevo siglo, donde es necesario incorporar consideraciones medioambientales en la planificación estratégica del mercadeo de una organización
(Dubey, 2008), siendo consecuencia de la relevancia que ha venido adquiriendo la problemática medioambiental en todas las empresas a nivel mundial. Estudios realizados evidencian que, aunque es un grado de integración que aún inicia por parte de las empresas, hay una tendencia al crecimiento y desarrollo de estrategias de mercadeo verde (Buil et al., 2009).

La Política Nacional Ambiental, enmarcada en el Proyecto Colectivo Ambiental del Plan Nacional de Desarrollo, buscaba la incorporación de las actividades ambientales de los sectores dinamizadores de la economía nacional, trabajando con el Ministerio del Medio Ambiente a través de la Dirección General Ambiental Sectorial y promoviendo estrategias de buenas prácticas ambientales encaminadas a la sostenibilidad ambiental y con ella la competitividad empresarial. Muestra de ello, ha sido la firma de un convenio entre el Ministerio del Medio Ambiente y la Sociedad de Agricultores de Colombia en el año 2000, con el decidido compromiso de los gremios afiliados a la SAC, las Corporaciones Autónomas Regionales, la academia y otras entidades relacionadas con la producción agropecuaria del país por trabajar en equipo el diseño y elaboración de guías ambientales para avanzar en acciones orientadas hacia el desarrollo sostenible, la protección del medio ambiente y el aprovechamiento racional de los recursos naturales (Asocolflores, 2002).

Desde la parte de la variable "Producto" con características ecológicas, las decisiones deben ir encaminadas a diseñar los atributos del producto, minimizando el consumo de recursos escasos y la generación de residuos a lo largo de los ciclos de vida del mismo, teniendo presente atributos específicos del producto como su duración, facilidad para reciclarse y/o reutilizarse, 
tipo y cantidad de materiales utilizados en el proceso de fabricación y generación de residuos. Con relación al componente "Precio", se debe reflejar la estructura de costos, incluidos los costos ecológicos derivados de su fabricación, y para la estrategia de competencia se debe tener en cuenta el VPC (Valor percibido por clientes y/o consumidores),

Con relación a la distribución, se requiere que el cliente o consumidor cuente con el producto en el lugar y momento oportuno, cantidad deseada y costo aceptable, fomentando igualmente aspectos como la redistribución (devolución y reciclado de envases), referente a "Promoción", esta debe educar y crear una imagen sobre responsabilidad ambiental. En los procesos de comunicación ecológica, los destinatarios no son únicamente el público objetivo sino todos los grupos de interés de la empresa, entidades gubernamentales, inversores, entidades financieras, asociaciones, entre otros. Es así que, una visión estratégica de la mezcla de marketing ecológico permite la satisfacción de las necesidades de clientes, el aumento de los beneficios de la empresa y la disminución de los impactos negativos sobre el medio ambiente (Hernández y López, 2012).

Cuando se habla de branding se toma en cuenta el nivel de marca, acorde al grado de opinión colectiva y su percepción dentro del mercado, permitiendo permanecer dentro de una determinada categoría, pero más allá de este concepto se requiere profundizar sobre este concepto como sustentable, el cual busca generar posicionamiento a través de una gestión de la marca, sobre su preocupación frente a su entorno y al ambiente, logrando equilibrio entre sociedad, economía y medio ambiente.

A pesar que las marcas cada día más se preocupan por lograr posicionamiento, estas no se preocupen por saber el destino final de sus envases, productos, publicidad e impresos utilizados en sus estrategias y tácticas de posicionamiento; por tanto, seguirán siendo marcas llamadas "Greenwashing", es decir, marcas que confunden al consumidor al hacerlo pensar, a través de argumentos ecológicos dentro de sus campañas publicitarias, puntos de venta y productos, que son marcas "amigables al medio ambiente", cuando realmente son altamente contaminantes o presentan prácticas de producción (Hernández, 2012).

Entonces, surge la pregunta ¿cuál es la necesidad del mercadeo verde?, siendo esta una amplia gama de ideas y aplicaciones, las cuales incluyen tecnología verde y tendencias para estimular ideas favorables para el medio ambiente. Así, una marca con conciencia verde se convierte en una alternativa viable y de percepción positiva frente a clientes y consumidores, denominando estos bienes como productos verdes, porque dentro de sus características se puede decir que son reciclables, reutilizables, biodegradables, con ingredientes naturales, químicos no tóxicos, contenidos bajo de químicos aprobados, sin deterioro para el medio ambiente, con envase, empaque y embalaje ecológico. Los productos verdes requieren de material renovable y reciclable lo que puede ser costoso, situación que clientes y consumidores desconocen y no estarían dispuestos a pagar más por un tipo de producto (Nagalakshmi, 2016).

Se ha logrado identificar que el green marketing es una herramienta para la protección del medio ambiente para las futuras generaciones, donde se ha influenciado en el comportamiento del consumidor inspirándole a comprar productos respetuosos con el ecosistema. Es evidente que, a partir de los resultados obtenidos, estos han aumentado, pero algunos aún carecen de consciencia "verde". Por lo tanto, una de las principales iniciativas tiene que ver con la necesidad de aumentar la conciencia entre el cliente, con respecto a los productos y su preocupación por el entorno. La sensibilidad 
de los precios muestra que los productos ecológicos son seguros y asequibles y los consumidores también están dispuestos a pagar el precio de estos beneficios (Thapa y Verma, 2014).

\section{Resultados y Discusión}

Como parte de los resultados, se puede observar que los floricultores de la Sabana de Bogotá están adelantando diversas actividades relacionadas con el mercadeo verde. Es así que, en el sector floricultor existe el programa Finca Flor Verde, una iniciativa de la Asociación Colombiana Exportadora de Flores (Asocolflores), cuyo objetivo es fortalecer las capacidades en las empresas floriculturas, desarrollando ventajas competitivas, responsables, sostenibles e innovadoras, mediante el acompañamiento a empresarios, permitiendo la implementación de mejores prácticas socio laborales, ambientales y de calidad en los cultivos. Este programa aborda temas relacionados con manejo de suelos, uso responsable de fertilizantes, manejo integrado de residuos, reducción y uso responsable de plaguicidas, conservación de la biodiversidad y el uso eficiente de energía; y va dirigido a productores afiliados a la Asociación de forma gratuita. Así, a través de 'La Ruta de la Tingua', se busca acompañar al productor en la implementación de las buenas prácticas ambientales, sociales y de calidad en sus fincas (Asocolflores, 2017).

Igualmente, en las empresas floricultoras de la Sabana de Bogotá se han presentado experiencias ambientales, como la encaminada a reducir el consumo de agua. Por medio de la reutilización de los lixiviados se buscaba recuperar los nutrientes perdidos con los vertimientos, reducir el consumo de agua y los costos de producción, además de minimizar los impactos al medio ambiente ocasionados por el consumo de agua y los nutrientes emitidos a los cuerpos de agua. Según Agromonte (2010), dicha iniciativa logró reutilizar los lixiviados de clavel, en los mismos claveles, desinfectando el agua, utilizando filtros lentos de arena y logrando la disminución inicial en el consumo de agua y fertilizantes de un 15\%, con un ahorro hasta del $30 \%$.

En la Figura 1 se muestra cómo, a pesar de estar esta práctica desde los años 80 , los floricultores de la población objeto de estudio desconocen técnicamente de su aplicación e implementación.

Por medio de las entrevistas, se logró identificar el desconocimiento del concepto de green marketing por parte de las empresas floricultoras

\section{Figura 1. Generalidades del Green Marketing}

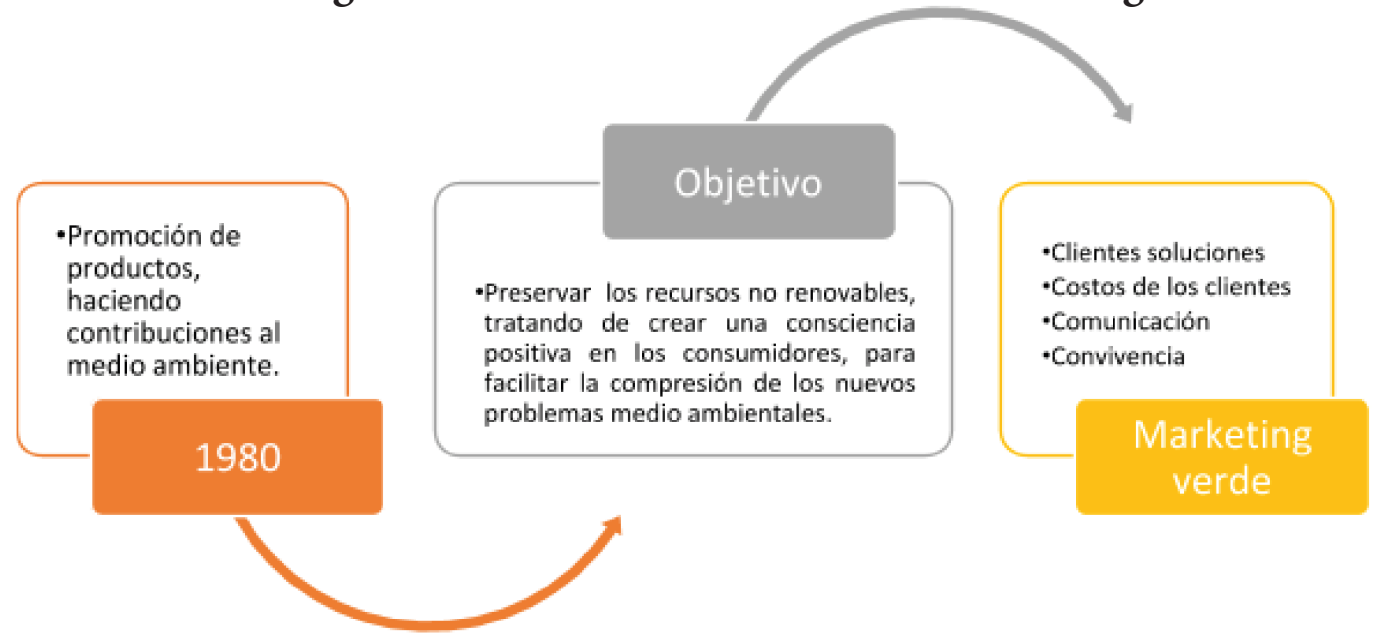

Fuente: Elaboración propia, 2018 
de la sabana de Bogotá pues, en ocasiones, se adelantan actividades consideradas parte de las estrategias encaminadas a desarrollar un mercadeo verde, que les permita mejorar su imagen y posicionamiento en el mercado, las cuales pueden ser incipientes como resultado del mismo desconocimiento y aplicación.

Siendo las flores un producto de exportación tradicional colombiano, como muchos otros, se identifica la necesidad de capacitación sobre la importancia no solo del concepto de mercadeo verde, sino el de ofrecer herramientas que permitan a los floricultores establecer un verdadero marketing verde en los diferentes procesos adelantados, que contribuyan a fortalecer la propuesta de valor, evidenciando y reflejando su compromiso con su entorno ambiental, y no como una "moda", sino como parte de la cultura empresarial.

\section{Figura 2. Actividades o procesos adelantados en la empresa floricultora para la protección del medio ambiente}

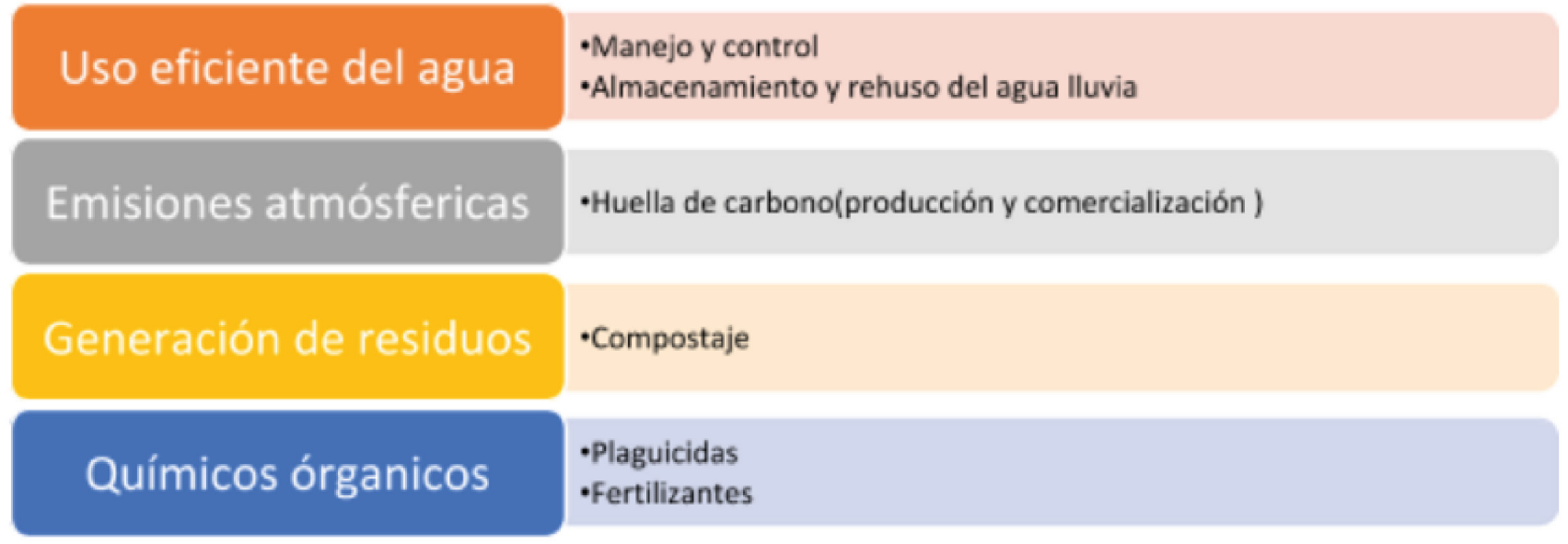

Fuente: Elaboración propia, 2018

La floricultura ha sido considerada una de las principales actividades económicas del país, con el aprovechamiento y uso adecuado de los sistemas de riego. Lo anterior está ligado a los costos de producción y apoyado en indicadores del programa Finca Flor Verde, permitiendo tener la información requerida por entidades gubernamentales y desarrollando estrategias de manejo del recurso hídrico que, permiten conocer el uso de agua superficial como subterránea, la utilización de aguas lluvias y el consumo en actividades de riego.
La producción de gases efecto invernadero, conocido como huella de carbono, generados por un producto o servicio durante su ciclo de vida (producción, procesamiento, transporte, venta detallada y uso), toma relevancia cuando se habla del cambio climático. Asocolflores (2002) realizó un estudio detallado del mercado nacional, siendo Colombia el segundo país exportador de flores, representada su producción en la sabana de Bogotá con el 76\%, la mayoría bajo el modelo de invernaderos. Los resultados mostraron que la huella de carbono para las rosas de corte se encuentra entre 0,61 y $2,30 \mathrm{~kg} \mathrm{CO} 2$ - eq por unidad funcional y la 
del clavel en un rango entre 0,59 y 1,11 kg CO2 eq por unidad funcional, considerando su proceso de producción, desde la extracción de materias primas hasta la puerta del avión. En ese orden de ideas, se toma como punto crítico el uso de los fertilizantes y el proceso de empaque y refrigeración, como en el proceso de descomposición de algunas flores incrementando emisiones de metano.

Como consecuencia de lo mencionado, nace la certificación Florverde, la cual trabaja en aspectos como manejo de aguas y riego, suelo y fertilizantes, manejo integrado de plagas, manejo integral de residuos y paisajismo y biodiversidad. De esta manera, las fincas certificadas disminuyen el uso de plaguicidas químicos en cerca de un $40 \%$, uso del agua superficial y subterránea en un 54\%, en los últimos 11 años (Córdoba, 2016).

Por otro lado, se pudo identificar la implementación de acciones por parte de los floricultores de la sabana de Bogotá con la utilización del compostaje como parte de su programa de manejo de residuos orgánicos, entendiéndose como un proceso de transformación de la materia orgánica para obtener compost -fertilizante compuesto de residuos orgánicos- y abono natural. Los abundantes desechos orgánicos vegetales por varias empresas floricultoras de la sabana de Bogotá está a cargo de terceros, por lo que se tiende a la creación de sitios para el desarrollo del compostaje, impulsado por el programa Florverde y Asocolflores, buscando mitigar el impacto ambiental y presentando falencias aún en aspectos relacionados con lixiviados, temperatura, olores, entre otros.

También se identificó la implementación de abonos orgánicos por parte de algunos productores de flores en la Sabana de Bogotá, y la disminución del plástico en sus procesos. Cabe destacar que los plaguicidas son elementos tóxicos, que se utilizan en las empresas floricultoras, muchos generados por requerimientos propios de mercados internacionales como nacionales, lo que requiere un manejo especial tanto por beneficio de los trabajadores como de la misma producción, evitando afectar a la población cercana.

Con la aparición de certificaciones del sector agrícola, se ha incentivado el no uso de plaguicidas prohibidos o restringidos en Colombia, logrando el aumento de productos biológicos, elaborados con materiales de origen biológico, tales como microorganismos, órganos y tejidos de origen vegetal o animal y biorracionales -sustancias que se derivan de microorganismos, plantas o minerales, de baja toxicidad para el ser humano-, tales como hongos, parasitoides, bacterias, entre otros, para el control de plagas y prevención de enfermedades en los floricultores.

Las flores de origen colombiano tienen una alta demanda a nivel internacional. Se identificó que además de atender el mercado local, los floricultores de la sabana atienden mercados internacionales en Norteamérica, Europa, Asia y Australia. Las exportaciones de flores desde el Departamento de Cundinamarca representaron el $80,5 \%$ para el año 2017 , siendo la rosa y el clavel los productos con mayor demanda en el mercado internacional (Dinero, 2017).

\section{Figura 3. Cobertura de mercado empresas floricultoras de la Sabana de Bogotá}

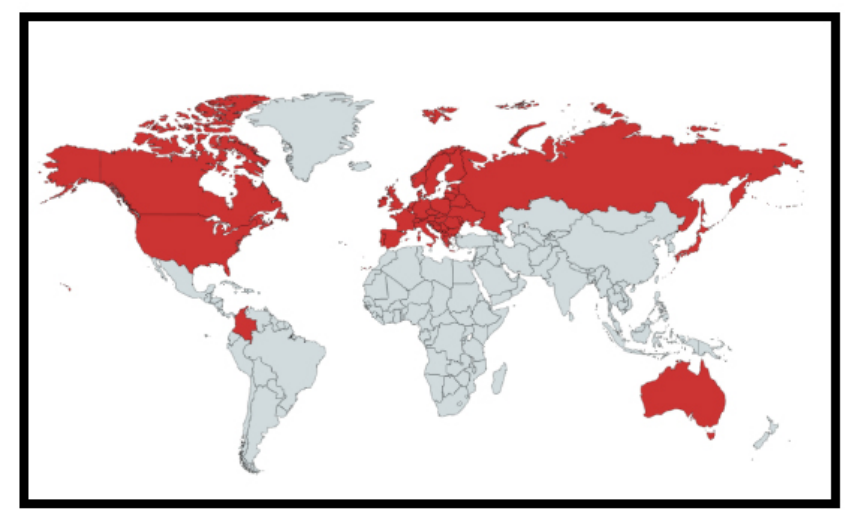

Fuente: Elaboración propia, 2018. 
Luego de identificar los mercados atendidos por los floricultores de la Sabana de Bogotá, se identificaron algunas certificaciones o requerimientos de los mercados objetivo, los cuales son una puerta de aceptación a algunos públicos, llevando a los empresarios del sector a trabajar por ser más competitivos y contribuir al desarrollo de procesos productivos y de comercialización, velando por la protección del medio ambiente y sirviendo como ventaja competitiva tanto en mercados de tipo nacional como internacional.

En Colombia, los floricultores trabajan por tener certificaciones como Florverde, otorgada por Asocolflores, como instrumento estratégico para promover la floricultura sostenible con responsabilidad social, con buenas prácticas agrícolas, minimización de agroquímicos, protección a los trabajadores, calidad del producto y responsabilidad gerencial. Así mismo, se presenta el Sello Ambiental Colombiano emitido por el Ministerio de Ambiente y Desarrollo Sostenible de Colombia, el cual busca consolidar la producción de bienes ambientalmente sostenibles e incrementar la oferta de servicios ecológicos competitivos en los mercados nacionales e internacionales; y la certificación Rainforest Alliance, que califica con altos criterios ambientales, sociales y económicos asociados con la agricultura sostenible.

Al indagar sobre estándares internacionales, los floricultores identificaron la norma 14001, como mecanismo para reconocer, priorizar y gestionar los riesgos ambientales, relacionados con su empresa; lo mismo que en mercados especiales como el alemán, con eco etiqueta "Ángel Azul”, símbolo de productos respetuosos con el medio ambiente, similar a la Etiqueta Ecológica Europea.

Se encontró también la certificación "Greal seal", del gobierno de los Estados Unidos, la cual establece criterios relacionados con el consumo de recursos renovables y energía, la contaminación atmosférica y de las aguas y la producción de residuos. "Environmental Choice Canada", eco etiqueta canadiense que certifica productos y servicios que ahorran energía, que utilizan material reciclado o que podrán reutilizarse. Un poco más lejos en el mercado australiano, se encuentra desde el mes de marzo del año 2018 requerimientos fitosanitarios establecidos por el Departamento de Agricultura y Recursos Hídricos, DAWR, dirigidos a evitar el ingreso de plagas cuarentenarias -especie, raza o biotipo vegetal o animal o agente patógeno dañino para las plantas o productos vegetales.

Con la norma internacional Global-Gap se da una certificación completa para la producción agropecuaria segura y sostenible, con el fin de beneficiar a los productores y consumidores en temas como medio ambiente, seguridad y salud en el trabajo, manejo de plagas, entre otros. Finalmente, es importante mencionar la certificación japonesa "Ecomark", la cual analiza las etapas del ciclo de vida del producto, teniendo en cuenta aspectos como el impacto y mejora ambiental al usarlo, así mismo los efectos ambientales relacionados con la generación de residuos.

\section{Figura 4. Certificaciones internacionales relacionadas con el sector floricultor}

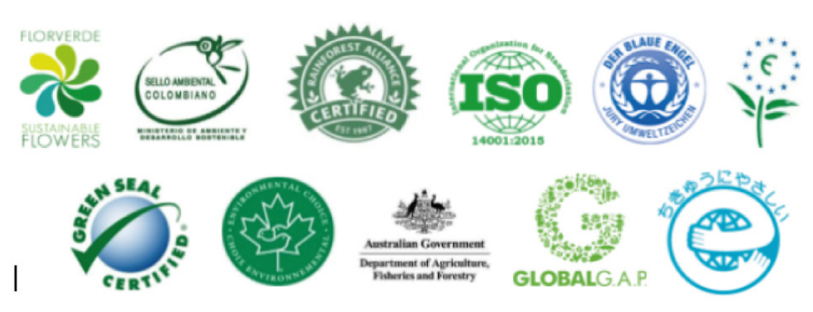

Aunque son varios los avances en los que trabajan las empresas floricultoras de la sabana de Bogotá, durante el trabajo de campo se pudo identificar que, a pesar de contar con procesos encaminados a desarrollar actividades de green marketing, no se establecen canales de difusión con los clientes, impidiendo la visibilidad de su compromiso con el medio ambiente, lo que puede redundar en valor diferenciador a sus productos, tanto en mercados nacionales como internacionales. Es- 
tos avances tampoco se destacan en el proceso de comercialización, pese a que se cuenta, en un alto porcentaje, con certificaciones o reconocimientos resultado del compromiso con el entorno; estos elementos contribuirían a generar mayor impacto y compromiso por parte de consumidores internos y externos al adquirir las flores.

\section{Conclusiones}

Se evidencia falta de conocimiento del concepto de green marketing dentro de las empresas floricultoras de la Sabana de Bogotá, debido a que es un concepto nuevo dentro del mercado floricultor. Sin embargo, es importante destacar que algunas empresas adelantan estrategias que buscan minimizar el impacto medio ambiental, así como en su proceso de distribución y comercialización. No obstante, el desconocimiento del manejo de este tipo de mercadeo verde no permite difundir dicho efecto como ventaja competitiva en mercados nacionales e internacionales.

Entre los principales beneficios de la implementación del green marketing se encuentra la maximización de recursos, lo que contribuye en la minimización de costos, debido al aprovechamiento de materiales que parecían inservibles, pero que por otro lado dan un valor agregado. Lo anterior, puede generar mayores ingresos, incrementando la demanda de productos comprometidos con el medio ambiente, resultado de la conciencia ambiental de clientes o consumidores, sin dejar de lado la buena imagen de la organización frente a su mercado objetivo.

La globalización hace que los productos tengan competencia no solamente a nivel local, sino internacional, con esto se requiere ir a la vanguardia de las exigencias de los diferentes mercados internacionales, donde el potencial comprador se encuentre interesado en el producto colombiano, en este caso para un producto insignia como las flores. Por tanto, al estar acompañado de certificaciones y reconocimientos que lo muestran como un producto comprometido con el medio ambiente, se accede a mercados altamente comprometidos con su protección.

El green marketing se convierte en una necesidad para las empresas, debido a la importancia de cuidar el medio ambiente, implementando medidas que contribuyen a crear consciencia ambiental, ofreciendo productos que además de satisfacer necesidades apoyen su conservación y mejoren la calidad de vida de las personas. Se recomienda hacer de este enfoque una parte ineludible de la cultura empresarial, garantizando un verdadero compromiso frente a la toma de decisiones, e involucrando a los diferentes miembros de la cadena de valor, desde los mismos proveedores, hasta el consumidor final.

\section{Referencias bibliográficas}

Agromonte. (2010). Reutilización de lixiviados de hidroponía en el cultivo de claveles. [en línea). Asocolflores, Editor, \& Asocolflores, Productor. [consulta: 23 agosto de 2017] Obtenido de: http://experiencias.florverde. org $/$ ?page_id $=86 \& \exp =94 \&$ tipo $=4$

Asocolflores. (2002). Guía Ambiental. [en línea]. Produmedios. [consulta: 15 septiembre de 2017]. Obtenido de: http://www.siame.gov. co/siame/documentos/Guias_Ambientales/ Gu\%C3\%ADas\%20Resoluci\%C3\%B3n\%20 $1023 \% 20$ del $\% 2028 \% 20$ de $\% 20$ julio\%20 de\%202005/AGRICOLA\%20Y\%20PECUARIO/Guia\%20ambiental\%20para\%20 el\%20subsector\%20Floricultor.pdf

Asocolflores. (2017). La ruta de la sostenibilidad. [consulta: 15 septiembre de 2017]. Recuperado de: http://www.rutadelatingua.org/

Berrio, S. (2006). Mercadeo verde: otra forma de ayudar al mundo [en línea]. [consulta: febrero 10 de 2017]. Recuperado de: https:// www.dinero.com/columna-del-lector/opin- 
ion/articulo/mercadeo-verde-otra-formaayudar-mundo/33121

Buil, I.; Fraj, E.; y Matute, J. (2009). La influencia del factor medioambiental en las estrategias de marketing: Un estudio aplicado al sector de bienes de consumo final. EsicMarket, 134, 115-180. [consulta: 12 noviembre de 2017]. Recuperado de: https://www.esic.edu/documentos/revistas/esicmk/090916_124542_E. pdf

Caguao, M. (2011). Mercadear con filosofía ecológica. Producto, (332), Venezuela (Pp. 46-50)

Córdoba, J. (2016): “ La gestión ambiental en la floricultura colombiana" [en línea] Revista de logística. [consulta: Septiembre 28 de 2018]. Recuperado de: https://revistadelogistica.com/actualidad/la-gestion-ambiental-en-la-floricultura-colombiana/

Chamorro, A. (2001): "El Marketing Ecológico" [en línea] 5campus.org, Medio Ambiente [consulta: Febrero 24 de 2018]. Recuperado de: http://www.5campus.org/leccion/ecomarketing

Darse. (s.f). 10 beneficios de implementar el Marketing Verde en una empresa. noticias.infocif.es [en línea]. [consulta: 10 de Septiembre de 2017]. Recuperado de: http://www.epu. edu.pe/darse/10-beneficios-de-implementar-el-marketing-verde-en-una-empresa/

Dinero. (2017). Colombia exportó US\$785 millones en flores al mundo en el primer semestre [en línea]. Revista Dinero [consulta 2 octubre 2018]. Recuperado de: https:// www.dinero.com/economia/articulo/exportaciones-de-flores-primer-semestre-de2017-en-colombia/248676
Dubey, P. (2008). Recycling Businesses: Cases of Strategic Choice for Green Marketing in Japan. IIMB Management Review (Indian Institute of Management Bangalore), 20 (3), India (Pp. 263-278).

Echeverry, L. (2010). Inserción del mercadeo verde en prácticas empresariales en Colombia (casos de estudio) [en línea]. Revista Luna Azúl. Vol 31, pp 122-138 [consulta 15 febrero 2016]. Recuperado de: http://www. redalyc.org/html/3217/321727233010/

González, O. y González, J. (2007). Enfoque de marketing y proactividad medioambiental. Mediterráneo económico, (11), España (Pp. 129-146)

Hernández Sampieri, R., Fernández Collado, C. y Baptista, L. (2010). Metodología de la Investigación. México: Mc Graw Hill. (P 80).

Hernández, W. R. (2012). Branding Sustentable. [en línea]. Revista del centro de investigación de la Universidad de la Salle 10, 95-99. [consulta: 15 septiembre de 2017]. Recuperado de: http://200.10.243.34/ojs/index.php/ rci/article/view/100/60

Hernández, Y., y López, D. (2012). El marketing ecológico y su integración en la planeación estratégica. Revista de estudios interdisciplinarios en ciencias sociales, 14(2), 223-231. [consulta: 15 septiembre de 2017]. Obtenido de: https://dialnet.unirioja.es/descarga/articulo/3990486.pdf

Marinao, E. y Valencia, V. (2012). Marketing Ecológico: Más que una moda, una herramienta competitiva. Revista Trend Management, Chile (Pp. 150-156)

Ministerio de Ambiente, Vivienda y Desarrollo Territorial. (2006). Lineamientos y recomendaciones para el programa social y am- 
biental flor verde en el marco de las evaluaciones Ambientales estratégicas según metodología del Departamento Nacional de Planeación [en línea]. (Documento). Ministerio de Ambiente, Vivienda y Desarrollo Territorial. [consulta 20 agosto de 2017]. Recuperado de: http://www.minambiente. gov.co/images/AsuntosambientalesySectorialyUrbana/pdf/Evaluaci\%C3\%B3n_Ambiental_Estrategica/Lineamientos__recomendaciones_flor_verde.pdf

Nagalakshmi, G. S. (2016). Impact of green marketing on consumer buying decision. [en línea]. International journal of research in commerce \& management, 7, 79-81. [consulta: 25 noviembre de 2017]. Obtenido de http://web.b.ebscohost.com/ehost/pdfview$\mathrm{er} / \mathrm{pdfviewer} ? \mathrm{vid}=2 \& \mathrm{sid}=\mathrm{ea} 260 \mathrm{~b} 13-7 \mathrm{~d} 9 \mathrm{a}-$ 4980-94ba-6f5f1b958867\%40sessionmgr103

Paternina, D. (2011). El marketing percibido desde lo ambiental [en línea]. Dictamen libre. Vol. 9. Pp. 13-16. [consulta: 24 noviembre de 2017]. Recuperado de: http://www.unilibrebaq.edu.co/ojsinvestigacion/index.php/ dictamenlibre/article/view/481/456

Palazón, P. (2009). El verde de moda. Producto (309), Venezuela (Pp. 34-36)

Pennano, C., y Díaz Artola, C. (2014). El marketing verde y su aporte al medio ambiente. Semana económica.com [en línea]. [consulta:
16 Febrero 2018]. Recuperado de: http:// semanaeconomica.com/article/economia/ medio-ambiente/149339-el-marketingverde-y-su-aporte-al-medio-ambiente/

Salgado Beltrán, L., Subirá Lopera, M., Beltrán Morales, L. (2009). Consumo orgánico y conciencia ambiental de los consumidores [en línea]. Problemas del desarrollo, Vol. 40, pp. 157, 189-199. [consulta: marzo 28 de 2018]. Recuperado de: http://www.revistas.unam. mx/index.php/pde/article/view/7763/7238

Sixto García, J., y Salgueiro Santiso, M. (2008). Comunicar el marketing verde y re-valorar la marca en las concienciadas [en línea]. XXXI Congresso Brasileiro de Ciências da Comunicação). Intercom - Sociedade Brasileira de Estudos Interdisciplinares da Comunicação, Natal. [consulta: 15 Octubre de 2017]. Recuperado de: http://www.intercom.org. br/papers/nacionais/2008/resumos/R31228-1.pdf

Thapa, S., y Verma, S. (2014). Analysis of green marketing as environment protection tool: a study of consumer of Dehradun. [en línea]. Internacional journal of research in commerce \& management, 9, 78-84. [consulta: 25 noviembre de 2017]. Recuperado de: http://www.ijre.org/wp-content/ uploads/2014/08/3.-analysis-of-green-marketing-as-environment-protection-tool.pdf 